\title{
Probing the quark-gluon plasma with a new Fermionic correlator
}

\author{
R. V. Gavail and Sourendu Guptal \\ Department of Theoretical Physics, Tata Institute of Fundamental Research, \\ Homi Bhabha Road, Mumbai 400005, India.
}

\begin{abstract}
We present the first measurement of a new correlation function of Fermion bilinears in finite temperature QCD with and without dynamical quarks in a quantum number channel in which nontrivial correlations are known to be present for purely gluonic operators. We find that the Fermion correlator vanishes for $T \geq 3 T_{c} / 2$, in agreement with the expectation for weakly interacting quarks in a quark-gluon plasma.
\end{abstract}

11.15.Ha, 12.38.Mh

hep-lat/9906018, TIFR/TH/99-29

The Relativistic Heavy Ion Collider (RHIC) in BNL, New York and the Large Hadron Collider (LHC) in CERN, Geneva may yield a new state of matter, called Quark-Gluon Plasma (QGP), which could have existed in our universe a few microseconds after the Big Bang. It is a theoretically challenging task to deduce from first principles as many properties of the plasma as possible. Such a program may help in devising clear and unique signals of QGP.

Lattice simulations of Quantum Chromo Dynamics (QCD) have provided a robust approach based on first principles towards this end. Such simulations of field theories in equilibrium at finite temperature $(T)$ use a discretisation of the Euclidean formulation for partition functions-

$$
Z(\beta)=\int \mathcal{D} \phi \exp \left[-\int_{0}^{1 / T} d t \int d^{3} x \mathcal{L}(\phi)\right],
$$

where $\phi$ is a generic field, $\mathcal{L}$ the Lagrangian density, and the Euclidean "time" runs from 0 to $1 / T$. The path integral is over field configurations which are periodic (anti-periodic) in Euclidean time for the Bosonic gluon (Fermionic quark) fields. Due to a lack of symmetry between the space and Euclidean time directions in eq. (1), this problem has only a subgroup of the full 4-dimensional rotational symmetry of the $T=0 \mathrm{Eu}$ clidean theory. Since the partition function above contains equal weights for all configurations which are related by these symmetries, only those operators which transform as scalars under this reduced symmetry group have non-vanishing expectation values.

For the lattice discretised problem the symmetry groups reduce to discrete subgroups of the continuum symmetry groups. It is useful to write the partition function of eq. (11) as the trace of the transfer matrix in one of the spatial directions. Correlation functions along that direction can then be classified by the irreducible representations (irreps) of the symmetry group of the transfer matrix. Unlike operator expectation values, correlation functions are generally non-vanishing in all representations - not just the scalar. At $T=0$, the symmetry group of the transfer matrix for QCD using the staggered Fermions for quarks has been studied extensively, and the representations of corresponding Fermion bilinear correlation functions are well known [3]. The symmetries and representations of screening correlation functions at finite temperature have been worked out recently 顿.

The main point of this last analysis is that the symmetry group of the $T>0$ transfer matrix is smaller than that of the $T=0$ transfer matrix. As a result, the $T=0$ irreps reduce further at finite temperature. All correlation functions block diagonalise under the isometry group of the spatial slice of the thermal lattice, the dihedral group $D_{4}^{h}$. As an example, a correlation function in, say, the $z$-direction of any vector $(\mathrm{V})$ or pseudo-vector $(\mathrm{PV})$ operator, $\left(V_{x}, V_{y}, V_{t}\right)$, in the $T=0$ theory breaks up into two scalar $\left(A_{1}^{+}\right)$irreps of $D_{4}^{h}$, the components $V_{t}$ and $V_{x}+V_{y}$, and a $B_{1}^{+}$irrep $V_{x}-V_{y}$. This happens for gluonic Wilson-loop operators such as plaquettes, and also for the quark bilinears operators 沺.

The plaquette operators, restricted to a $z$-slice, transform as a PV set at $T=0$ and provide a good example of this reduction. The combinations $P_{x y}$ and $P_{t x}+P_{t y}$ transform as the $A_{1}^{+}$(scalar) component of the PV and have non-vanishing expectation values at finite temperature. On the other hand, $P_{t x}-P_{t y}$ transforms as the $B_{1}^{+}$[5]. Due to the reasons given earlier, this last expectation value must vanish, and we show later that it does. However, the correlation function need not, and, indeed, does not. Non-trivial screening has been observed through gauge-invariant gluonic correlation functions [5] in all the other quantum number channels (labelled by the irreps of $D_{4}^{h}$ ) as well.

Screening masses obtained from correlation functions built out of staggered Fermion field operators have also been extensively studied in the past [6] 8]. The correlators which have been measured in the past are the $A_{1}^{+}$ from the scalar (S) and pseudo-scalar (PS) channels, and $A_{1}^{+}$combinations of the vector $(\mathrm{V})$ and pseudo-vector (PV) channels. The two $A_{1}^{+}$correlators descending from $\mathrm{S}$ and PS see a lower screening mass $(\mu)$ than those descending from the $\mathrm{V}$ and $\mathrm{PV}$. The latter are consistent with the expectation from free Fermion field theory- 


$$
\mu a=2 \sinh ^{-1}\left(\sqrt{(m a)^{2}+\sin ^{2}\left(\frac{\pi}{N_{t}}\right)}\right),
$$

where $a$ is the lattice spacing, $m$ the quark mass, and $N_{t}$ is the number of lattice sites in the Euclidean time direction $\left(T=1 / N_{t} a\right)$. Even some other measurements, such as those of "wavefunctions", which seemed to indicate a more complicated picture [9], can be understood in terms of weakly interacting quarks [10]. Here we re-examine the screening masses with the complete decomposition of operators into irreps of the finite temperature invariance group. In particular, we report in this letter the results of the first measurement of the $B_{1}^{+}$correlation function constructed from local Fermion bilinears (see Table III of 伍) and compare our results with those obtained with gluonic operators.

We have simulated QCD with four light degenerate flavors of quarks at temperatures above the phase transition temperature, $T_{c}$, on lattices of size $4 \times 10^{2} \times 16$, using the Hybrid Monte Carlo (HMC) algorithm 11]. The longest direction, $N_{z}$, was chosen to be four times the Euclidean time direction, $N_{t}$, so that correlations could be followed to a distance of $2 / T$. One simulation was performed at $T=3 T_{c} / 2$ with the coupling $\beta=5.1$ and the quark mass $m=0.015 / a$ where $a$ is the lattice spacing. The second simulation was made at $T=2 T_{c}$ with $\beta=5.15$ and $m=0.01 / a$. With our choice of $N_{t}=4, a=1 / 4 T$. The temperature identifications are made using previous measurements of the critical coupling on lattices with larger values of $N_{t}$ [12]. Companion runs were made in quenched $\mathrm{QCD}$ on lattices of the same size at couplings corresponding to $3 T_{c} / 2$ and $2 T_{c}$ for the quenched theory using a Cabbibo-Marinari pseudo-heat-bath algorithm 13.

We thermalised the HMC simulation at $3 T_{c} / 2$ with two different runs - one starting from an ordered gauge configuration, and the other from a pure gauge configuration thermalised at $2 T_{c}$. Agreement in measurements of all thermodynamic quantities was used to decide on thermalisation. The plaquette average turned out to be the most stringent test, since it is the least noisy. At $2 T_{c}$ thermalisation was tested by checking that a run starting from an ordered gauge configuration gave the same thermodynamics as one starting from a thermal $3 T_{c} / 2$ configuration.

Once thermalisation was achieved, two runs were made at each temperature - one with a trajectory length of one molecular dynamics (MD) time unit, and another with a trajectory length half as long. At $3 T_{c} / 2$ statistics were collected from 875 such configurations generated using the long trajectory, and 285 with the short trajectory. Previous studies have shown that the physics is much simpler at $2 T_{c}$. We did smaller runs at this temperature - collecting statistics of 445 configurations with the short trajectory length and 100 with the long trajectories.
The question of autocorrelations is important whenever statistical inferences are to be made. It was found that autocorrelations of local operators, such as plaquettes, were the same with the two different trajectory lengths mentioned above. However, with any simulation algorithm that undergoes critical slowing down, short distance operators are decorrelated faster than those which are dominated by large distance scales. Thus, the effective number of measurements of short distance operators is not the same as that for extended operators. This is most problematic for correlation function measurements, where the correlator at different distances may have entirely different autocorrelations. These are usually difficult to measure directly and a different approach to the problem seems necessary.

If the errors in Fermion bilinear correlation functions, $\Delta C(z)$, are evaluated with the assumption that there are no autocorrelations, then they depend systematically on the separation $z$. We found that for sufficiently large statistics, $\Delta C(z)$ falls exponentially with $z$ and its logarithmic slope is almost independent of statistics. Hence it is possible to quote a single number as a figure of merit for decorrelation-

$$
D=\frac{\Delta C\left(N_{z} / 2\right)}{\Delta C(0)} .
$$

$D$ is usually larger than unity, and depends on the specifics of the simulation algorithm and its tuning. Since smaller values of $D$ are preferable, tuning the algorithm should be done to minimise this.

The values of $D$ obtained depended on the channel being studied: the largest values of $D$ were found in the $A_{1}^{+}$ irreps coming from the $\mathrm{V}$ or the $\mathrm{PV}$, and the smallest in the $B_{1}^{+}$irreps. In each channel, we found only a weak dependence of $D$ on the number of measurements - indicating that it is a direct measure of the efficiency of the algorithm.

We found that the long trajectories (1 MD time unit) give about half the value of $D$ as obtained with the shorter trajectory. Since it takes twice as long to run the longer trajectory, the computational effort, $E=$ $D \times(C P U$ time $)$, involved in getting equal statistical errors is the same for these two trajectory lengths. However, the analysis of correlated errors in screening mass measurements is simplified with the longer trajectories. In test runs with trajectories 2.5 MD time units long, we found no further decrease in $D$, and hence an increase in $E$. Such a dependence of $E$ on trajectory length is characteristic of the HMC simulation algorithm [14]. In the dynamical QCD simulations, we found $D$ to lie in the range 250 to 1000 . The quenched simulations were significantly easier to decorrelate - the value of $D$ was lower by a factor of roughly 40 compared to the full theory simulations. This information on autocorrelations has been incorporated in all our statistical analyses. 
In Table I, we report our measurements of screening masses for the known $A_{1}^{+}$correlators. Our results are in very good agreement with previous measurements in the quenched theory at $N_{t}=4$ [8]. There is also a remarkable agreement between the $A_{1}^{+}$screening masses obtained from the quenched and the dynamical Fermions simulations at both temperatures. The four $A_{1}^{+}$irreps coming from the $\mathrm{V}$ and $\mathrm{PV}$ channels gave degenerate screening masses which agree extremely well with the free field theory estimate in eq. (2). The $A_{1}^{+}$correlators in the $\mathrm{S}$ and PS channels gave smaller screening masses, which increase marginally with $T$.

Hadron mass measurements in 4-flavor QCD at quark mass, $m a=0.01$ and $\beta=5.15$, corresponding to our runs at $2 T_{c}$, have been performed before 15. A comparison with these $T=0$ measurements, listed in the last column of Table I, shows that our finite temperature screening masses are completely different-

$$
\frac{\mu(T)}{m(T=0)} \approx \begin{cases}3 & \left(A_{1}^{+} \text {from PS }\right), \\ 2 & \left(A_{1}^{+} \text {from }\right) .\end{cases}
$$

In contrast, earlier measurements for $N_{t}=8$ lattices yielded $\mu / m \approx 1$ for the $A_{1}^{+}$screening mass in the vector channel V [7]. This made it difficult to argue for a thermal effect, although $\mu$ agreed with eq. (2) even in that case. The present measurement resolves this problem.

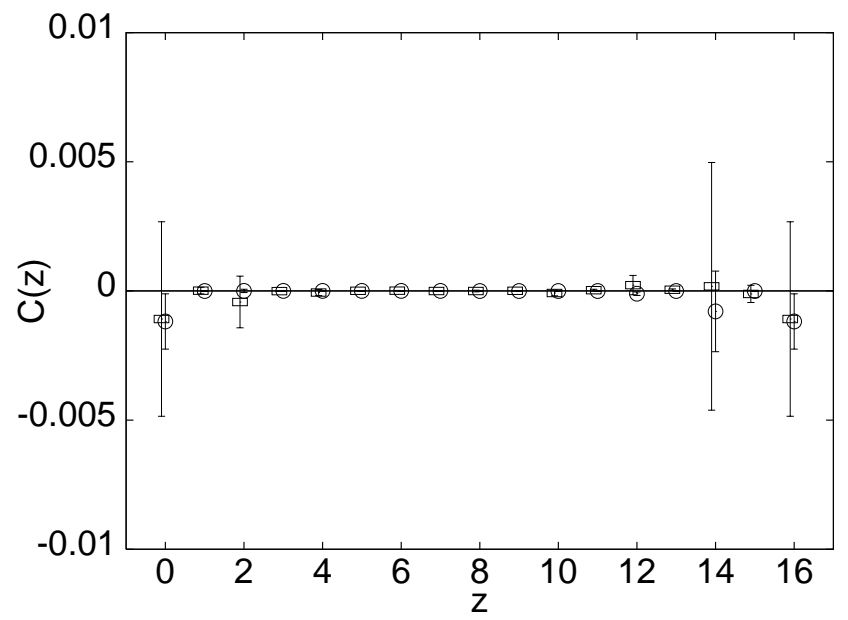

FIG. 1. The $B_{1}^{+}$correlation function obtained from the $\mathrm{V}$ channel operators. Boxes denote data for dynamical QCD simulations for $3 T_{c} / 2$ and circles for $2 T_{c}$.

Our main new result is the first measurement of the correlation function in the $B_{1}^{+}$channels. Correlators in this irrep obtained with $\mathrm{PV}$ and $\mathrm{V}$ are identical up to a sign, configuration by configuration. Hence we restrict our attention only to the $B_{1}^{+}$coming from the $\mathrm{V}$. We found that the $B_{1}^{+}$correlation functions vanish to the best of the measurement precision (see Figure 1). The correct statistical procedure is to quote the $\chi^{2}$ value for the fit of the data by a correlation function which is identically zero. We found

$$
\chi^{2} / \mathrm{DOF}= \begin{cases}7 / 15 & \left(\frac{3}{2} T_{c}, \text { dynamical }\right), \\ 11 / 15 & \left(2 T_{c}, \text { dynamical }\right) .\end{cases}
$$

The quenched runs gave very similar results-

$$
\chi^{2} / \mathrm{DOF}= \begin{cases}7 / 15 & \left(\frac{3}{2} T_{c}, \text { quenched }\right) \\ 5 / 15 & \left(2 T_{c}, \text { quenched }\right)\end{cases}
$$

The numbers in eq. (5) have been obtained with the statistics collected in the longer of the two runs made at each temperature. The HMC simulations with smaller statistics also gave very similar results at both these temperatures.

One possible explanation for these remarkable results is an exact symmetry between the $x$ and $y$ directions, configuration by configuration. If this were so, then nonscalar operators would vanish, not only on the average, but identically. As a result, the correlation function in all but the scalar channel would also vanish.

We test for this symmetry by correlation functions of the $B_{1}^{+}$plaquette operator, discussed earlier. Its average must vanish, and does-

$$
\left\langle P_{t x}-P_{t y}\right\rangle= \begin{cases}(-0.6 \pm 1.8) \times 10^{-4} & \left(\frac{3}{2} T_{c}, \text { quenched }\right), \\ (5.5 \pm 3.8) \times 10^{-5} & \left(2 T_{c}, \text { quenched }\right) .\end{cases}
$$

However, the corresponding correlation function does not vanish. The same configurations used in the Fermionic correlator measurements lead to gluonic $B_{1}^{+}$correlations which are clearly non-zero.

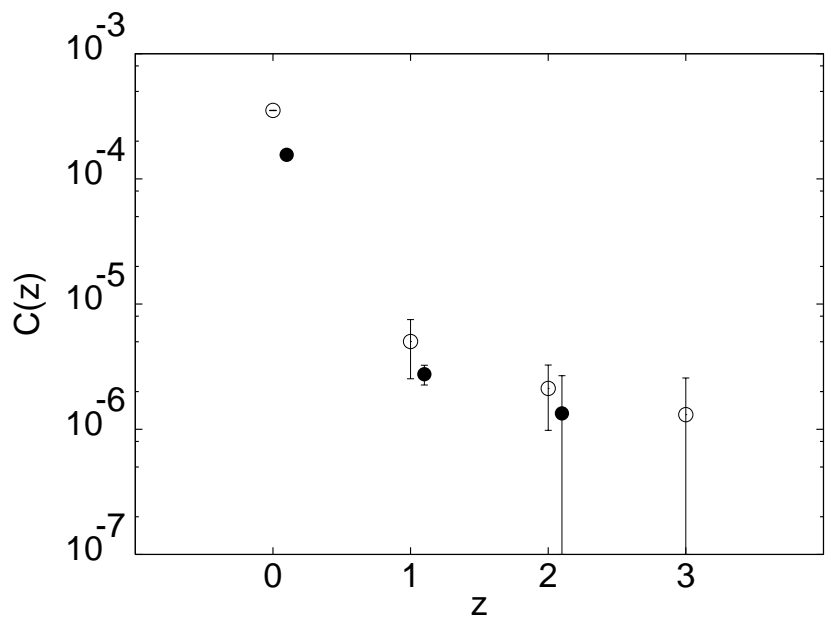

FIG. 2. The $B_{1}^{+}$correlation function obtained from the plaquette operators. Filled circles denote data for $3 T_{c} / 2$ and open for $2 T_{c}$.

This correlation function is exhibited in Figure 2 for the quenched QCD simulations. Since the corresponding screening mass is large [5], the correlation function is somewhat noisy at large distances but it is clearly very different from zero at small distances. The test 
of a vanishing value of this correlation function gives $\chi^{2} / \mathrm{DOF} \approx 800$. Thus the gauge configurations underlying our measurements have a strong configuration-toconfiguration asymmetry between the $x$ and $y$ directions, allowing the gluonic $B_{1}^{+}$correlators to survive.

Another possibility is that we have accidentally chosen an operator which has small overlap with the lowest $B_{1}^{+}$ eigenvector of the transfer matrix. One way to test this is to work with other operators. It is often the case that the overlap on the lowest eigenvector improves by delocalising the operator in some way. We constructed the "meson" operators using quark propagators computed with fuzzed links. This fails to improve the $B_{1}^{+}$correlation function. We conclude that there must be a dynamical reason for the vanishing of the $B_{1}^{+}$correlator in the high temperature phase of QCD, when measured using Fermion bilinears.

The numerical agreement of the screening masses extracted from the exponential fall-off of the $A_{1}^{+}$-correlators with free field values of eq. (2) has been used earlier to argue that one sees weakly interacting quarks in the high temperature phase of QCD. In the free-field theory limit, the Fermionic $B_{1}^{+}$correlators studied here would vanish. Consequently, our observations can be seen as additional evidence for the weakly interacting picture. Indeed, comparing the $\chi^{2}$ values, we find that the $B_{1}^{+}$effective coupling in the quark sector is approximately 40 times smaller than that in the gluon sector, assuming the overlaps to be similar. Since the vanishing or existence of a correlation function is easy to observe, we believe that the $B_{1}^{+}$correlator is a qualitatively better indicator of the non-interacting nature of the quarks in the quark-gluon plasma.

The drawback of this picture of non-interacting Fermions is well-known - the $A_{1}^{+}$irreps coming from the $\mathrm{S}$ and PS channels are not degenerate with those coming from the $\mathrm{V}$ and $\mathrm{PV}$ channels. A plausible argument to understand this phenomenon is to note that the $A_{1}^{+}$correlator coming from the $\mathrm{S}$ channel mixes with the glue sector of the theory. As a result, the screening mass in this channel will be contaminated by those in the gluonic $A_{1}^{+}$sector, of which the lowest is the Debye screening mass, $m_{D}$. In quenched simulations at $3 T_{c} / 2$, $m_{D} / T=2.8 \pm 0.2$ [5]. Since the screening mass for the $\mathrm{S}$ channel lies in between $m_{D}$ and the $\mathrm{V}$ channel mass, as seen in Table If, it is consistent with such a conjecture.

We employed staggered Fermions for this investigation. It would be interesting to confirm these results for the Wilson Fermions as well. The symmetries of the lattice are, of course, independent of the type of Fermions employed and the group theoretic arguments apply with small modifications. In particular, the break-up of the zero temperature spectrum under the $D_{4}^{h}$ group proceeds without change, although the actual operators realising the irreps do change.
To summarize, we have used a new Fermionic correlator to demonstrate that the quarks in the quark-gluon plasma are weakly interacting already at temperatures as low as $3 T_{c} / 2$. The particular correlator we used is a much better probe of Fermion interaction strength than those used earlier.

[1] Electronic mail: gavai@tifr.res.in

[2] Electronic mail: sgupta@tifr.res.in

[3] H. Kluberg-Stern et al., Nucl. Phys., B 220 (1983) 447; M. F. L. Golterman and J. Smit, Nucl. Phys., B 255 (1985) 328; M. F. L. Golterman, Nucl. Phys., B 273 (1986) 663.

[4] S. Gupta, e-print hep-lat/9903019, Phys. Rev. D in press.

[5] B. Grossmann et al., Nucl. Phys., B 417 (1994) 289; S. Datta and Sourendu Gupta, Nucl. Phys., B 534 (1998) 392.

[6] C. DeTar and J. Kogut, Phys. Rev. Lett., 59 (1987) 399; Phys. Rev., D 36 (1987) 2828; S. Gottlieb, et al., Phys. Rev. Lett., 59 (1987) 1881; A. Gocksch, P. Rossi and U. M. Heller, Phys. Lett., B 205 (1988) 334; G. Boyd, S. Gupta and F. Karsch, Nucl. Phys., B 385 (1992) 481. T. Hashimoto, T. Nakamura and I. O. Stamatescu, Nucl. Phys., B 400 (1993) 267; Ph. de Forcrand et al., e-print hep-lat/9901017.

[7] K. Born, et al., (The $M T_{c}$ collaboration), Phys. Rev. Lett., 67 (1991) 302.

[8] S. Gupta, Phys. Lett., B 288 (1992) 171.

[9] C. Bernard, et al., Phys. Rev. Lett., 68 (1992) 2125.

[10] T. H. Hansson and I. Zahed, Nucl. Phys., B 374 (1992) 277; V. Koch et al., D 46 (1992) 3169.

[11] S. Duane, A. D. Kennedy, B. J. Pendleton and D. Roweth, Phys. Lett. , B 195 (1987) 216.

[12] F. R. Brown, et al., Phys. Lett., B 221 (1990) 181; R. V. Gavai, et al., (The $M T_{c}$ collaboration), Phys. Lett., B 241 (1990) 567.

[13] N. Cabibbo and E. Marinari, Phys. Lett., 119 B (1982) 387.

[14] S. Gupta, Nucl. Phys., B 370 (1992) 741.

[15] R. Altmeyer et al., (The $M T_{c}$ collaboration), Nucl. Phys., B 389 (1993) 445.

\begin{tabular}{cccc}
\hline \hline$\overline{\text { Channel }}$ & $3 T_{c} / 2$ & $2 T_{c}$ & $T=0$ \\
\hline $\mathrm{S} A_{1}^{+}$ & $0.91(3)$ & $1.07(4)$ & $0.60(5)$ \\
$\mathrm{PS} A_{1}^{+}$ & $0.91(3)$ & $1.07(4)$ & $0.303(2)$ \\
$\mathrm{V} A_{1}^{+}$ & $1.39(7)$ & $1.35(6)$ & $0.71(6)$ \\
$\mathrm{PV} A_{1}^{+}$ & $1.39(7)$ & $1.35(6)$ & $1.4(1)$ \\
\hline \hline
\end{tabular}

TABLE I. Screening masses in units of the lattice cutoff, $1 / a=4 T$. The $T=0$ masses quoted here were measured 15 with the same lattice spacing and quark mass as the runs at $2 T_{c}$. 